\title{
The DAC system and associations with acute leukemias and myelodysplastic syndromes
}

\author{
Gesine Bug • Oliver G. Ottmann
}

Received: 10 November 2010 / Accepted: 12 November 2010/Published online: 14 December 2010

(C) The Author(s) 2010. This article is published with open access at Springerlink.com

\begin{abstract}
Summary Imbalances of histone acetyltransferase (HAT) and deacetylase activity (DAC) that result in deregulated gene expression are commonly observed in leukemias. These alterations provide the basis for novel therapeutic approaches that target the epigenetic mechanisms implicated in leukemogenesis. As the acetylation status of histones has been linked to transcriptional regulation of genes involved particularly in differentiation and apoptosis, DAC inhibitors (DACi) have attracted considerable attention for treatment of hematologic malignancies. DACi encompass a structurally diverse family of compounds that are being explored as single agents as well as in combination with chemotherapeutic drugs, small molecule inhibitors of signaling pathways and hypomethylating agents. While DACi have shown clear evidence of activity in acute myeloid leukemia, myelodysplastic syndromes and lymphoid malignancies, their precise role in treatment of these different entities remain to be elucidated. Successful development of these compounds as elements of novel targeted treatment strategies for leukemia will require that clinical studies be performed in conjunction with translational research including efforts to identify predictive biomarkers.
\end{abstract}

Keywords Epigenetic therapy - Deacetylase inhibitors . Myelodysplastic syndromes · Acute myeloid leukemia . Drug development $\cdot$ Targeted therapy $\cdot$ Hypomethylating agents

G. Bug • O. G. Ottmann $(\bowtie)$

Medizinische Klinik II, Abteilung für Hämatologie und Onkologie der, Goethe-Universität,

Theodor-Stern-Kai 7,

60590, Frankfurt, Germany

e-mail: ottmann@em.uni-frankfurt.de

\section{Histone deacetylases as therapeutic targets in hematologic malignancies}

Among the epigenetic modifications associated with the development of human cancer, alterations of the acetylation status of histones play a prominent role (see [1] for review). The extent of acetylation and deacetylation on different positions of core histones is determined by the antagonistic activity of histone acetylases (HAT) and histone deacetylases (HDAC) and alters the nucleosomal conformation of both transformed and non-transformed cells. Deacetylation of histones by HDACs hinders the accessibility of DNA to transcription factors that are involved in determining malignant cell behaviour, thereby changing their activity, subcellular localisation and interaction partners. In addition, acetylation is an important post-translational modulation of a wide range of nuclear and cytoplasmic proteins involved in the regulation of a multitude of cellular functions (e.g., p53, tubulin, heat-shock protein 90). A disrupted equilibrium between HDACs and HATs, with preponderance of deacetylase activity, leads to transcriptional repression of a diverse set of genes involved in the regulation of cell proliferation, differentiation and apoptosis. Aberrant gene transcription caused by abnormal activity of HATs and HDACs is commonly observed in leukemia and lymphoma [2-6]. Accordingly, modulation of protein lysine acetylation through inhibition of histone deacetylases (HDACs) is currently being considered as an attractive new therapeutic strategy for acute myeloid and lymphoid leukemias and myelodysplastic syndromes. 


\section{Involvement of HDACs in myeloid malignancies (AML and MDS)}

\section{Aberrant HDAC activity in acute leukemia}

In acute myeloid and lymphoid leukemia, recurring chromosomal translocations result in the generation of chimeric fusion genes, many of which function as transcriptional regulators. Several of these fusion proteins are associated with the development of leukemia, partly by causing transcriptional deregulation of genes responsible for differentiation and inhibition of apoptosis through mechanisms linked to chromatin alterations. Binding of oncogenic transcription factors such as PML-RAR $\alpha$, PLZFRAR $\alpha$, AML1-ETO, and others, induce deposition of aberrant chromatin marks by recruiting histone deacetylases (HDACs), histone methyltransferases (HMTases), and DNA methyltransferases (DNMTs) to target genes [7, 8].

Chimeric fusion oncoproteins in leukemia may also undergo inappropriate forced dimerization [7,9] which alters the association between the DNA-binding portion in the chimeric oncoproteins and their transcriptional cofactors. This mechanism has been particularly well studied in the core binding factor leukemias, in which the fusion proteins resulting from the chromosomal translocations $t$ $(8 ; 21)(\mathrm{q} 22 ; \mathrm{q} 22)$ (AML1-ETO) and inv(16)(p13q22) (CBFB-MyH11) act as transcriptional repressors [7, 8]. These fusion proteins are characterized by the disruption of $\mathrm{CBF}$, a heterodimeric transcription factor that is important in hematopoietic differentiation. CBF consists of the DNA binding component, AML1 (also known as RUNX1 or CBFA2), and CBFB, which stabilizes AML1. Wild-type AML1 functions as a transcriptional activator. In contrast, in CBF leukemias the dimerization domains of the partners of AML1, which include TEL, ETO, and MTG16, repress the transcription of AML1 target genes by either recruiting HDACs directly or by recruiting co-repressor-HDAC complexes such as $\mathrm{N}-\mathrm{CoR} / \mathrm{Sin} 3 / \mathrm{HDAC} 1$ to AML1 responsive promoters. This keeps the associated histones in the deacetylated state, thereby repressing normal AML1 target genes involved in differentiation and cell cycle inhibition [7-11]. AML1-ETO also directly represses transcription of tumour suppressor genes such as ARF through the AML1 DNA binding domain [12]. HDACs have also been found in complexes with proteins that regulate cell cycle checkpoints such as $\mathrm{Rb}$ and its family members [13]. Recently, AML1-ETO has also been shown to recruit DNA methyltransferase 1 (DNMT1) [14] This finding implies that transcriptional silencing of AML1 target genes occurs at least partly through an interplay between histone deacetylation and promoter DNA methylation.

Collectively, these mechanistic insights support the rationale for developing inhibitors of HDAC activity, which, by correcting the transcriptional deregulation of genes involved in cell cycle regulation and apoptosis, could have therapeutic value.

Disruption of HATs in acute leukemia

In addition to the recruitment of HDACs, DNMTs and corepressor complexes, epigenetic deregulation in acute leukemias and myelodysplastic syndromes also includes a disruption of HATs by gene mutations or chromosomal translocations. Two closely related HATs, E1A-associated $300 \mathrm{kDa}$ protein $(\mathrm{p} 300)$ and the CREB binding protein (CBP), play distinct but essential roles in hematopoiesis, e. g. as co-activators for several transcription factors including p53, pRB, MYB, JUN, FOS, and RUNX1 [15]. CBP/p300 are regarded as functional tumor suppressors, for mice with inactivated alleles of these gene loci develop hematological malignancies [16].

Cancer-associated mutations abrogating the enzymatic activity of HATs have been observed. CBP/p300 are located on chromosomes 16p13 and 22q13, respectively; regions, which are frequently lost in tumors [17-19]. In addition, rearrangement of both genes has been shown in chromosomal translocations, resulting in aberrant gain or loss of acetylation activity. For example, the $\mathrm{t}(11 ; 16)(\mathrm{q} 23 ; \mathrm{p} 13)$ translocation produces mixed lymphcyte leukemia (MLL)CBP fusion proteins. Unlike wild-type MLL, these fusion proteins cause aberrant acetylation at the HOX loci, which in turn up-regulates the expression of HOX genes and causes the subsequent development of leukemia. A similar mechanism may apply to MLL-p300 fusion proteins derived from the $\mathrm{t}(11 ; 22)(\mathrm{q} 23 ; \mathrm{q} 13)$ translocations $[11,15$, 20].

The $\mathrm{t}(8 ; 16)(\mathrm{p} 11 ; \mathrm{p} 13)$ translocation leads to the production of Monocytic leukaemia zinc finger protein (MOZ)-CBP fusion proteins. MOZ and monocytic leukemia zinc finger protein-related factor (MORF) belong to the MYST family of HATs. In normal hematopoietic cells, MOZ functions as a transcriptional co-activator to potentiate RUNX1-dependent gene expression and stimulate cell differentiation. On contrary, MOZ-CBP fusion proteins down-modulate RUNX1dependent gene expression and thus lead to leukemogenesis. A similar mechanism may apply to other chromosomal abnormalities involving MOZ and MORF genes, such as inversion of chromosome $8(\operatorname{inv}(8))$ which fuses the HAT domain of MOZ to the transcription factor TIF2 (also known as NCOA2). TIF2 is a member of the p160 family of nuclear receptor coactivators known to interact with p300/CBP. MOZ-TIF2 fusion results in the deregulation of transcription through the aberrant recruitment of CBP to nucleosomal regions targeted by MOZ $[10,15]$. 


\section{Epigenetic alterations in acute lymphoblastic leukemia}

Transcriptional silencing of genes due to epigenetic mechanisms is an important alteration in acute lymphoblastic leukemia (ALL), with research so far having focused on the role of DNA methylation. An exception is the $t(12 ; 21)$ (p13;q22) (TEL-AML1), which is restricted to precursor Bcell lineage leukemia and is the most common $(\sim 25 \%)$ translocation in childhood acute lymphoblastic leukemia (ALL). Like AML1 in the CBF acute myeloid leukemias, the abnormal TEL-AML1 fusion protein can bind to core enhancer sequences. Instead of activating transcription through recruitment of co-activators and HATs, it recruits co-repressors and HDACs. Since TEL-AML1-induced transcriptional repression was shown to be reversed by HDAC inhibitors, TEL-AML1-positive ALL may be considered likely to benefit from treatment with HDAC inhibitors [21, 22] Preclinical studies testing this hypothesis are reviewed elsewhere in this paper.

Down-regulation of microRNAs (miRNA) by epigenetic mechanisms, most notably by DNA methylation, may contribute to tumorigenesis. A recent study explores the epigenetic alterations of miRNAs in ALL by analyzing the methylation and chromatin status of the miR-124a loci in ALL [23]. Expression of miR-124a was down-regulated in ALL by hypermethylation of the promoter and histone modifications including decreased levels of acetylated histone H3. Epigenetic down-regulation of miR-124a induced an up-regulation of its target, cyclin-dependent kinase 6 (CDK6) as well as phosphorylation of retinoblastoma $(\mathrm{Rb})$, and contributed to the abnormal proliferation of ALL cells both in vitro and in vivo. CDK6 inhibition by inhibition of HDACs decreased ALL cell growth in vitro. Although an analysis of 353 patients diagnosed with ALL revealed an association between a higher relapse and mortality rate and hypermethylation of the tumor suppressor microRNA Hsa-miR-124a, these results nevertheless provide the rationale for therapeutic strategies in ALL that either target the epigenetic regulation of microRNAs and/or directly target the CDK6-Rb pathway, e.g. by deacetylase inhibitors.

\section{DAC inhibitors in hematologic malignancies: molecular targets and biochemical / pharmacologic properties}

At present, 18 HDAC isoforms are known which are grouped into four classes. From a functional as well as from a translational point of view, class I HDACs (isoforms $1,2,3,8)$ are the best characterized proteins of this family. Less extensive information is available on function and expression of class II isoforms $[4-7,9,10]$ while little is known about class III HDACs (the sirtuins) and HDAC11, which on the basis of distinct structural properties has been suggested to constitute an HDAC class on its own.

A large variety of well established as well as novel HDAC inhibitors possess antineoplastic activity in vitro and in animal models in vivo. Some of these inhibitors are unselective, i.e. they target class I and II but not class III, while others target only specific HDAC classes or isoforms. As these compounds also inhibit deacetylation of numerous non-histone proteins, they should preferably be referred to more broadly as deacetylase inhibitors (DACi). DACi may be subdivided into two categories, which inhibit nuclear and cytoplasmic deacetylases, respectively.

The chemical structure of DACi encompasses three subunits: (1) a zinc-chelating group, (2) a usually hydrophobic "spacer" group, and (3) a catalytic domain which determines the specificity of the compound. DACi inhibitors are classified by structure and include the short-chain fatty acids valproic acid and sodium butyrate, the cyclic tetrapeptides romidepsin (depsipeptide, FK228, FR901228), Trapoxin A, and Apicidin, the hydroxamic acids vorinostat (suberoylanilide hydroxamic acid, SAHA), trichostatin A (TSA), LAQ824, panobinostat (LBH529) and PXD101, the benzamides MS-275, CI994, und MGCD-0103, cyclic tetrapeptides, electrophilic ketones (trifluoromethylketone), and others (depudecin, SNDX-275, and isothiocyanates) [4]

Although these DACi differ in structure, potency and possibly HDAC enzyme selectivity, they target primarily class I and II HDACs and do not affect the activity of the class III sirtuins (reviewed by Marchion and Münster [24]) It is becoming increasingly clear that the downstream effects of HDAC inhibition, which ultimately leads to growth inhibition and apoptosis in different tumor types, depend upon both the HDAC inhibitor and the cell type [25-27]. Only very recently, research began to focus on the histone acetylation status in human tumours in general and the specific expression of HDAC isoforms in solid as well as hematological malignancies.

Non-histone targets of DACi in acute myeloid leukemia

HATs and DACs affect the acetylation status of lysine residues not only of histones but also of transcription factors (TF) (eg, p53, E2F1, GATA1, RelA, Y1, MAD/ MAX, TFIIE and TFIIF, and hormone receptors). Altered acetylation of TFs may affect their DNA binding and transcriptional activity [4-6, 28]. In addition, DACs have been shown to deacetylate a multitude of proteins other than histones or transcription factors, e.g. the cytoskeleton protein-tubulin, $\beta$-catenin, DNA repair enzymes and the heat shock protein 90 (hsp90) [29-35]. Accordingly, modulation of DAC function by DACi may not only affect gene transcription but also modify the stability of 
proteins, as well as the ability of proteins to interact with DNA and other proteins involved in important biologic functions in the leukemic cells [36]. The sheer number of transcription factors known to be acetylated suggests that the acetylation of these nonhistone proteins may have as much regulatory effect on transcription as the acetylation of histone proteins. A more comprehensive description of nonhistone targets of acetylation has been reviewed recently [36-38].

The molecular basis for the relatively selective antitumor activity of DACi is unknown. Insigna et al. investigated the effects of DACis on leukemias expressing PML-RAR or AML1-ETO [39]. Even though these oncoproteins are known to initiate leukemogenesis through deregulation of HDACs, it was shown that oncogene expression is not sufficient to confer DACi sensitivity to normal cells. DACiinduced induction of apoptosis in leukemic cells was found to be p53 independent and depend upon activation of the death receptor pathway (TRAIL and Fas signaling pathways). Interestingly, TRAIL, DR5, FasL and Fas were upregulated by DACis in the leukemic cells, but not in normal hematopoietic progenitors, indicating that sensitivity in leukemias is a property of the fully transformed phenotype and depends on DACis-induced activation of a specific death pathway.

DACi-mediated induction of TRAIL was also identified as a mediator of the selective anticancer action by Nebbioso et al. [40]. Expression of TRAIL, by directly activating the TNFSF10 promoter, triggered tumor-selective death signaling in acute myeloid leukemia (AML) cells, without inducing apoptosis in normal CD34(+) progenitor cells. DACi induced proliferation arrest, TRAIL-mediated apoptosis and suppression of AML blast clonogenicity occurred irrespective of karyotype.

Heat shock proteins (HSPs) are molecular chaperones that stabilize folding and conformation of both normal and oncogenic proteins. These chaperones thereby prevent the formation of protein aggregates. HSPs are often overexpressed in human malignancies, including AML (see [41] for review), and are the main chaperones required for the stabilization of multiple oncogenic kinases involved in the development of AML. HSP90 client proteins are involved in the regulation of apoptosis, proliferation, autophagy and cell cycle progression; several of these proteins are considered possible therapeutic targets for treatment of AML. The results from initial phase I/II clinical trials testing HSP90 inhibitors have documented that HSP90 inhibition can mediate antileukemic effects in vivo. HSP90 activity is also regulated by posttranscriptional modulation, and HSP90 inhibition can thereby be indirectly achieved through increased acetylation caused by DACi (see section Combination with other agents)

\section{Preclinical studies of DAC inhibitors in acute leukemias}

Single-agent DACi in AML

Valproic acid (VPA) has a long history of use as an antiepileptic drug. After in vitro studies demonstrated that VPA induced differentiation of carcinoma cells and leukemic blasts derived from patients with AML, it was discovered that VPA acts as a DACi [42]. In addition, VPA causes selective proteasomal degradation of HDAC2, but not of other class I HDACs (i.e., HDAC 1, 3, and 8) [42].

In $\mathrm{t}(8 ; 21)$ acute myeloid leukemia (AML), the AML1/ ETO fusion protein promotes leukemogenesis by recruiting class I HDAC-containing repressor complex to the promoter of AML1 target genes, as described above. VPA disrupts the physical interaction between AML1/ETO and HDAC1, stimulates the global dissociation of the AML1/ETOHDAC1 complex from the promoter of AML1/ETO target genes, and induces relocation of both the AML1/ETO and HDAC1 proteins from the nucleus to a perinuclear region. Mechanistically, these effects are associated with a significant inhibition of HDAC activity, histone $\mathrm{H} 3$ and $\mathrm{H} 4$ hyperacetylation, and recruitment of RNA polymerase II, resulting in transcriptional reactivation of target genes (i.e., IL-3) otherwise silenced by the AML1/ETO fusion protein. Ultimately, these pharmacological effects resulted in significant antileukemic activity mediated by partial cell differentiation and caspase-dependent apoptosis [43].

VPA was recently shown to enhance proliferation and self-renewal of normal hematopoietic stem cells, raising the possibility that VPA may also support growth of leukemic progenitor cells (LPC). Indeed, VPA maintained a significantly higher proportion of CD34(+) LPC and colony forming units compared to control cultures in six AML samples, but selectively reduced leukemic cell numbers in another AML sample with expression of AML1/ETO. These data suggest a differential effect of VPA on the small population of AML progenitor cells and the bulk of aberrantly differentiated blasts in the majority of AML samples tested [44].

The new hydroxamic acid derivative, ITF2357, blocked proliferation and induced apoptosis in AML1/ETO-positive Kasumi-1 and primary blast cells in concentration of $0.1 \mu \mathrm{M}$, whereas AML1/ETO-negative HL60, THP1 and NB4 cell lines were sensitive only to $1 \mu \mathrm{M}$ ITF2357. In Kasumi-1 cells, ITF2357 induced AML1/ETO degradation through a caspase-dependent mechanism and also determined DNMT1 efflux from, and p300 influx to, the nucleus. Moreover, ITF2357 induced local H4 acetylation and release of DNMT1, HDAC1 and AML1/ETO, paralleled by recruitment of p300 to the IL-3 gene promoter. ITF2357 treatment, however, did not induce re-expression of IL-3 gene. Accordingly, the methylation level of IL-3 
promoter, as well as of several other genes, was unmodified [45].

As VPA and ITF2357 seem to specifically target AML1/ ETO-driven leukemogenesis, integration of both class Iselective and pan-DACi in novel therapeutic approaches for AML1/ETO-positive AML may be worthwhile.

Single-agent DACi in acute lymphoblastic leukemia

The potential anti-leukemic activity of DACi in ALL has been examined in a small number of preclinical studies; most of these tested DACi as a single agent in vitro using human ALL cell lines as models.

An early study compared the in vitro effects of the cyclic tetrapeptide romidepsin (FK228) on human leukemia/ lymphoma cells and cell lines with normal hematopoietic cells [46]. Romidepsin induced G1 arrest and / or apoptotic cell death at nanomolar concentrations. Clinical samples from patients with ALL were more sensitive to romidepsin at clinically achievable drug concentrations than either normal peripheral blood or bone marrow mononuclear cells or normal progenitor cells. Expression levels of HDAC-1 and HDAC-3 proteins did not correlate with the sensitivity to romidepsin.

The anti-leukemic activity and mode of action of the hydroxamic acid derivative, LAQ824 was studied using four human pre-B lymphoblastic cell lines as models representing different cytogenetic subsets (Sup-B15 and TMD-5, both $\mathrm{t}(9 ; 22)$ positive, SEM, $\mathrm{t}(4 ; 11)$ positive, and NALM-6 cells). LAQ824 significantly inhibited the proliferation of leukemic lymphoblastic cell lines; this was due to increased apoptosis accompanied by activation of caspase- 3 and caspase-9, cleavage of poly(ADP-ribose)-polymerase (PARP) as well as by down-regulation of Bcl-2 and disruption of the mitochondrial membrane potential. Surprisingly, LAQ824-induced apoptosis was partially independent of caspase activation [47].

Panobinostat (LBH589), a broad-spectrum DACi closely related to the hydroxamate LAQ824 but with more favorable pharmacologic properties, potently induced cell-cycle arrest, apoptosis, and histone (H3K9 and H4K8) hyperacetylation in two human cell line models of Philadelphia chromosomenegative acute lymphoblastic leukemia (T-cell MOLT-4 and pre-B-cell Reh). Panobinostat treatment increased mRNA levels of proapoptosis-, growth arrest-, and DNA damage repair genes including FANCG, FOXO3A, GADD45A, and GADD45B. This was associated with increased histone acetylation at the GADD45G promoter and phosphorylation of histone H2AX. Treatment with panobinostat also induced apoptosis in primary ALL cells, including those from a relapsed patient [48].

A comparison of the growth inhibitory and apoptotic activity of several DACi's that differ in their subclass selectivity towards glucocorticoid sensitive and resistant acute lymphoblastic leukemia cells (ALL) and patient blasts was reported by Tsapis et al. [49]. Vorinostat (SAHA) displayed similar efficacy on glucocorticoid-sensitive and insensitive ALL cells but did not synergize with dexamethasone. Both B-precursor and T-ALL cells were much more efficiently killed by VPA and by the pan-DACi vorinostat than by the sub-class I selective DAC inhibitor MS275. This group's previous observation that MS275 and vorinostat displayed a similar growth inhibitory and proapoptotic activity in the AML cell line U937 suggest that cellular context may be an important determinant of the biologic response to DACi. The mechanisms of vorinostat-induced apoptosis in ALL likewise were cell-line dependent, acting through initiator caspase 10 in CEM-C7 cells while inducing apoptosis through the intrinsic, as well as through caspase-independent death pathways in CEM-C1 cells. In addition, vorinostat induced re-expression of DR5 in glucocorticoid-insensitive CEM-C1 cells that do not express DR5 and are insensitive to TRAIL, indicating that vorinostat may overcome glucocorticoid resistance by inducing alternative death pathways. This ability of DACi to induce apoptosis in glucocorticoid-resistant ALL was confirmed in a more recent report [49]. Taken together, these studies establish that several DACi possess potent growth inhibitory activity against B-precursor and T-cell ALL largely independent of specific cytogenetic aberrations such as TEL-AML1 [50]. Anti-leukemic activity is associated with up-regulation of genes critical for DNA damage response and growth arrest. These findings are not restricted to the in vitro setting, as DACi have demonstrated potent antitumor activity in two NOD/ SCID mouse models of B-cell precursor childhood ALL [51].

Combination of DACi with differentiation-inducing agents in AML and MDS

Treatment with DACi's alone or in combination with either all-trans retinoic acid (ATRA) or granulocyte colonystimulating factor is able to overcome inhibition of differentiation caused by chimeric fusion oncoproteins such as PMLRAR $\alpha$, PLZF-RAR $\alpha$, or AML-ETO [5, 52-55]. In addition, combination of DACi's with ATRA induces differentiation of APL cells that are resistant to treatment with ATRA alone [54]. Recently, primary leukemia blasts were shown to undergo differentiation following treatment with a combination of ATRA and DACi's [42, 55].

Combination with hypomethylating agents

Functionally, histone acetylation and DNA methylation are tightly linked. For example, DNA methyltransferases 
(DNMTs) direct different HDACs to specific sections of chromatin B-precursor and T-cell with repressed genes and thereby augment gene repression. Since DNA methylation is the principal mechanism for suppressing gene expression, methylated genes are typically resistant to treatment with DACi alone. However, sequential exposure of malignant cells with a DNMT-inhibitor followed by a DACi had synergistic effects on reactivation of gene expression and induction of apoptosis [56, 57].

The anti-apoptotic transcription factor NFkappaB is constitutively activated in myeloblasts arising in high-risk myelodysplastic syndromes (MDS) and AML. Recent studies indicate that DNMT inhibitors (such as azacytidine and decitabine) and DACi (such as trichostatin A and VPA) can inhibit the constitutive activation of NFkappaB in malignant myeloblasts in vitro and in vivo. The rapid onset of this effect within a few hours suggested that it was not due to epigenetic reprogramming; indeed, DNMT and HDAC inhibitors reduced the phosphorylation of the NFkappaB-activating kinase IKK $\alpha / \beta$. Thus, inhibition of NFkappaB and IKK $\alpha / \beta$ by DNMT inhibitors in concert with DACi may contribute to the antileukemic activity of these classes of agents [58].

Combined treatment with panobinostat and decitabine may potentiate antileukemia activity against human AML cells by other mechanisms as well. The histone methyltransferase EZH2 modulates DNA methylation by recruiting DNMT1 to the DNA. Exposure to the pan-DACi panobinostat reduces DNMT1 and EZH2 protein levels by inhibiting the chaperone association of Hsp90 with DNMT1 and EZH2. This promotes their proteasomal degradation and disrupts the interaction of DNMT1 with EZH2. In K562 myeloid leukemia cells, co-treatment with panobinostat and decitabine caused greater diminution of DNMT1 and EZH2 levels than either agent alone. Exposure to panobinostat and decitabine in combination augmented induction of apoptosis of primary AML but not normal CD34(+) bone marrow progenitor cells and may thus exerts clinically relevant antileukemia activity against human AML cells [59].

\section{Combination of DACi with chemotherapy}

Molecular perturbations resulting from exposure to DACi lower the threshold for apoptosis by multiple mechanisms. This supports the rationale for combining DACi with conventional cytotoxic agents, which generally trigger apoptosis by perturbing cell cycle and/or inducing DNA damage [28]. Additional support for this basic concept stems from studies in solid tumors, demonstrating that cotreatment with DACi, e.g. vorinostat or LAQ824, significantly enhances the cytotoxic effects of docetaxel, epothilone B, and gemcitabine against human breast cancer cells
[34], of fluorouracil against colorectal cancer and of etoposide, camptothecin, doxorubicin, and cisplatinum against glioblastoma cells $[60,61]$. Co-treatment with phenylbutyrate has also been documented to increase the activity of conventional chemotherapeutic agents commonly used in treatment of acute leukemias such as cytarabine and etoposide, against non-Hodgkin's lymphoma, chronic lymphoid leukemia (CLL), and multiple myeloma cells $[62,63]$.

The potential value of combining DACi with topoisomerase II-inhibitors is based on preclinical studies suggesting a DACi-mediated stimulation of the enzyme in human leukemic cells [64, 65]. Anthracyclines induce DNA double strand breaks by forming a complex with both the DNA and the DNA repair enzyme topoisomerase II, which disrupts DNA replication and transcription [66].

The pan-DACi panobinostat was tested in combination with doxorubicin on AML cell lines and primary leukemia blasts and induced cell death by an increase in the mitochondrial outer membrane permeability and release of cytochrome $\mathrm{c}$ from the mitochondria, resulting in caspasedependent apoptosis accompanied by upregulation of Bax, Bak and Bad. The drug combination provoked a strong activation of a DNA damage response, indicating that this combination may trigger cell death by a mechanism that induces DNA double-strand breaks [67]. These data are consistent with a report by Sanchez et al, which showed that simultaneous exposure of AML cells to idarubicin and VPA or vorinostat in vitro led to a significant and synergistic increase in the proportion of apoptotic cells [66]. Taken together, these data indicate that the combination of panobinostat and an anthracycline may be an effective therapy for the treatment of AML.

Apart from anthracyclines, cytosine arabinoside (ara-C) and etoposide are the most widely used chemotherapeutic agents for treatment of AML. The DACi vorinostat was shown to induce topoisomerase II expression and sensitize human leukemia cells to etoposide and other inhibitors of topoisomerase II [65]. The combination of etoposide or ara$\mathrm{C}$ with vorinostat was evaluated in a preclinical study using cell proliferation assays and cell cycle analysis to determine the effects on HL-60 myeloid leukemia cells and K562 myeloid blast crisis chronic myelogenous leukemia cells. The sequence of drugs proved to be important: cytotoxic antagonism resulted when vorinostat was combined concomitantly with ara- $\mathrm{C}$, whereas the sequential administration with vorinostat given prior a drug-free interval followed by ara-C was mostly synergistic. Etoposide combined with vorinostat was additive to synergistic, and the synergism became more pronounced when etoposide was given after vorinostat. Cell cycle analyses revealed that the sequence-dependent interaction of vorinostat and ara-C or etoposide reflected the arrest of cells in G1 or G2 phase 
during vorinostat treatment and recovery into $\mathrm{S}$ phase after removal of vorinostat [68].

Interactions between the novel benzamide DACi MS275 and fludarabine were examined in lymphoid and myeloid leukemia cells in relation to mitochondrial injury, signal transduction events, and apoptosis. Prior exposure of Jurkat lymphoblastic leukemia cells to a marginally toxic concentration of MS-275 for 24 hours sharply increased mitochondrial injury, caspase activation, and apoptosis in response to a minimally toxic concentration of fludarabine, resulting in highly synergistic antileukemic interactions and loss of clonogenic survival. Simultaneous exposure to MS275 and fludarabine also led to synergistic effects, but these were not as pronounced as observed with sequential treatment. Similar interactions were noted with myeloid cell lines, other DACi (e.g., sodium butyrate) and other nucleoside analogues (gemcitabine). Potentiation of fludarabine lethality by MS-275 was associated with acetylation of histones $\mathrm{H} 3$ and H4, down-regulation of the antiapoptotic proteins XIAP and Mcl-1, enhanced cytosolic release of proapoptotic mitochondrial proteins (e.g., cytochrome c, Smac/DIABLO, and apoptosis-inducing factor), and caspase activation. It was also accompanied by the caspasedependent down-regulation of $\mathrm{p} 27$, cyclins $\mathrm{A}, \mathrm{E}$, and D1, and diminished phosphorylation of retinoblastoma protein. Combined exposure of cells to MS-275 and fludarabine was associated with a significant increase in generation of reactive oxygen species (ROS); moreover, both the increase in ROS and apoptosis were largely attenuated by coadministration of the free radical scavenger L-N-acetylcysteine [69].

The drugs used in ALL-specific regimens differ considerably from those employed in treatment of AML. The antifolate methotrexate (MTX), a universal component of ALL therapies, is metabolized by folylpoly-gammaglutamate synthetase (FPGS) into long-chain polyglutamates (MTX-PG(3-7)), resulting in enhanced cytotoxicity from prolonged inhibition of dihydrofolate reductase (DHFR) and thymidylate synthetase (TS). Identification of a hypersensitive site upstream from exon-1 of the FPGS gene suggested that chromatin remodeling could alter FPGS expression. Leclerc et al. demonstrated that HDAC1 is recruited by NFY and Sp1 transcription factors to the FPGS promoter in ALL cell lines. Exposure of these cell lines to the DACi's sodium butyrate and vorinostat increased FPGS mRNA expression by 2- to 5-fold, whereas DHFR and TS mRNA expression was decreased. Combination treatment with MTX plus vorinostat significantly enhanced cytotoxicity and apoptosis in B- and TALL cell lines as compared with each drug alone. Vorinostat lead to the intracellular accumulation of longchain MTX-PG(3-7). Therefore, DACi-induced FPGS expression increases the accumulation of MTX-PG(3-7) and cytotoxicity in ALL cell lines, which is potentiated by DHFR and TS downregulation [70].

Taken together, the findings indicate that DACi induces multiple perturbations in signal transduction, survival, and cell cycle regulatory pathways that lower the threshold for chemotherapy-mediated mitochondrial injury and apoptosis in human myeloid and lymphoid leukemia cells. These data also provide insights into possible mechanisms by which novel, clinically relevant DACi might be used to enhance the antileukemic activity of a wide spectrum of standard chemotherapeutic agents, including anthracyclines, nucleoside analogues such as fludarabine, topoisomerase inhibitors and antimetabolites. While these observations suggest that the combination of DACi and cytotoxic drugs may enhance the efficacy of therapy for AML and ALL, there is evidence that the sequence of drug administration may be of paramount importance, and that inattention to proper scheduling of these agents may even be antagonistic. Furthermore, the effect of combination treatment on nonhematologic organ toxicity is unknown. Other caveats include the possibility of drug-drug interactions and the theoretic potential of DACi's to induce drug resistance by a variety of mechanisms, as will be discussed below (see section "DACi-associated drug resistance"). Ultimately, well-controlled phase I and II studies will be necessary to determine the feasibility and clinical utility of these promising strategies.

\section{Combination with other agents}

Chimeric oncoproteins with constitutive tyrosine kinase activity such as BCR-ABL and mutant receptor tyrosine kinases such as mutant fms-like tyrosine kinase 3 (FLT3) contribute to the leukemic phenotype in a variety of acute and chronic leukemias and are therefore targeted clinically by selective inhibitors of the respective tyrosine kinases. In addition, these aberrant kinases are client proteins of the molecular chaperone heat shock protein 90 , raising the possibility that inhibition of HSP90 could lead to depletion of these oncogenic proteins and act synergistically with TKIs in mediating anti-leukemic effects. The pan-DACi LAQ824 induces acetylation and inhibition of HSP90. When biphenotypic leukemia MV4-11 cells were treated with LAQ824, levels of FLT-3 and p-FLT-3 were attenuated due to enhanced proteasomal degradation of these kinases. Cotreatment with LAQ824 and PKC412 synergistically induced apoptosis of MV4-11 cells and enhanced apoptosis of primary AML cells expressing mutant FLT-3. These data support the notion that the combination of LAQ824 and PKC412 is highly active against human AML cells with mutant FLT-3 [71].

The effect of the DACi MS-275 against a variety of human leukemia cells with defined genetic alterations was 
explored by Nishioka et al. [72]. MS-275 induced growth arrest of MOLM13 and MV4-11 AML cells, which possess internal tandem duplication mutation in the FLT3 gene (FLT3-ITD). Exposure of these cells to MS-275 decreased levels of total, as well as, phosphorylated forms of FLT3, resulting in inactivation of its downstream signal pathways, including Akt, ERK, and STAT5. Further studies found that MS-275 induced acetylation of Hsp90 in conjunction with ubiquitination of FLT3, leading to degradation of FLT3 proteins in these cells. The action of MS-275 in leukemia cells was potentiated by inhibition of MEK/ERK signaling, suggesting that DAC inhibition may be useful for treatment of individuals with leukemia possessing activating mutation of FLT3 gene.

An analogous experimental approach was taken in a study investigating the effect of MS-275 in combination with pharmacological blockage of Akt/mammalian target of rapamycin (mTOR) signaling in HL60 AML and NB4 acute promyelocytic leukemia (APL) cells [72]. Inactivation of mTOR by the rapamycin analog RAD001 (everolimus) significantly enhanced MS-275-mediated growth inhibition and apoptosis of these cells. When used in combination, RAD001 potentiated the ability of MS-275 to induce differentiation of HL60 and NB4 cells. Proliferation of HL60 tumor xenografts in nude mice was significantly inhibited by combining RAD001 with MS-275, without adverse effects. Taken together, concomitant administration of a DACi and an mTOR inhibitor may be a promising treatment strategy for the individuals with a subset of human leukemia.

Although VPA is a less potent DACi than the hydroxamic acid or benzamide derivatives, strong anti-proliferative and pro-apoptotic effects of VPA were observed on human ALL and CML cell lines at concentrations achievable in vivo. These effects were most pronounced in ALL cell lines as well as in primary ALL cells. Notably, VPA revealed enhanced activity with imatinib mesylate, nilotinib, the farnesyl transferase inhibitor SCH66336, interferon-alpha and cytosine arabinoside. VPA inhibited the growth of colony-forming cells from $12 \mathrm{Ph}+$ chronic-phase CML patients, but also of those from normal healthy controls in a dose-dependent fashion. In conclusion, VPA, whether alone or in combination with other non-classical anti-leukemic compounds, exerts significant anti-leukemic effects on human ALL and CML cells [73].

The proteasome has been successfully targeted for the treatment of multiple myeloma and mantle cell lymphoma, but proteasome inhibition alone has been clinically less effective in other hematologic malignancies. In leukemic cell lines, the novel proteasome inhibitor NPI-0052 and DACi synergistically induced apoptosis in leukemia cells in a caspase-8- and oxidant-dependent manner. In leukemic mice, treatment with NPI-0052 reduced the white blood cell (WBC) burden over 35 days. The combination of NPI-0052 with the DACi MS-275 or valproic acid was more effective than the combination of bortezomib with these DACi's [74]. Further studies aimed at identifying mechanisms of synergy revealed that NPI-0052 elicits caspase- 8 and oxidative stress-dependent epigenetic alterations, and that DAC inhibition repressed expression of the proteasomal beta5, beta2, and beta1 subunits, consequently inhibiting respective enzymatic activities. These results indicate that crosstalk between NPI-0052 and DACi contributes to the synergistic cytotoxic effect on leukemia cells. This reinforces the potential clinical utility of combination studies of DACi and proteasome inhibitors [75].

\section{Clinical experience with deacetylase inhibitors in MDS and AML}

To date, clinical examination of DACi in patients with AML has met with limited success. Current experience is mostly restricted to phase I or phase II studies involving small numbers of patients.

Valproic acid is the only DACi that has been clinically investigated in larger numbers of patients with MDS. An early clinical trial involving 18 MDS patients showed an overall response rate of 37\% (1 partial response (PR) and 6 hematologic improvements (HI)). All-trans retinoic acid (ATRA) did not enhance the clinical activity in patients who had not already demonstrated a response to VPA [76]. In a subsequent report of 43 patients with MDS, the overall response rate was $35 \%(1 \mathrm{PR}$, and $15 \mathrm{HI})$ [77]. Efficacy of the VPA +/- ATRA treatment seems to be inversely correlated with the stage of the disease. According to Kuendgen et al., low or intermediate-I IPSS scores or a normal bone marrow blast count proved to be good predictors for response [78].

The combination of VPA with ATRA was also tested in AML patients who were considered ineligible for intensive chemotherapy. A disappointingly low response rate of only 5\% with no CR was observed among 40 patients receiving this combination [79] Similarly, none of 26 patients with high-risk AML achieved a CR in another study examining combined treatment with VPA and ATRA [80]. Results did not improve when ATRA was added later on in another pilot study with 8 AML patients [81]. Nevertheless, 5 out of 11 patients with de novo AML responded to a therapy with VPA, ATRA and theophylline $(1 \mathrm{CR}, 2$ complete remissions with incomplete recovery of peripheral blood counts (CRi), $2 \mathrm{HI}$ ) [82].

In view of the development of the novel, considerably more potent DAC inhibitors vorinostat, panobinostat, romidepsin and the isotype-specific MGCD0103, the use of VPA in patients with AML, as a single agent or in 
combination with ATRA, does not appear very promising. The role of VPA in combination with hypomethylating agents will be addressed in a later section of this review.

Vorinostat was investigated as a single agent in a phase I study with 31 AML and 10 MDS patients. The maximal tolerated dose was $200 \mathrm{mg}$ BID. Seven patients experienced hematologic improvement, including $4 \mathrm{CR}$ in patients with AML. Increased histone acetylation was observed at all dose levels [83]. These favorable results were not confirmed in a randomized phase II trial, in which only one out of 37 AML patients achieved CR [84].

Panobinostat (LBH589) was administered in a phase I study as a 30-min infusion on days 1 to 7 of a 21-day cycle. Fifteen patients with AML $(n=13)$, ALL $(n=1)$, or MDS $(n=1)$ were treated. At doses $<11.5 \mathrm{mg} / \mathrm{m}^{2}$, i.v. panobinostat was well tolerated with consistent, albeit transient antileukemic and biologic effects: in 8 of 11 patients peripheral blasts declined, but rebounded following the 7day treatment period. The median acetylation of histones $\mathrm{H} 2 \mathrm{~B}$ and $\mathrm{H} 3$ in $\mathrm{CD} 34(+)$ and $\mathrm{CD} 19(+)$ cells increased significantly during therapy, as did apoptosis in CD14(+) cells [85]. Oral panobinostat was evaluated in patients with advanced hematologic malignancies. Doses of $\geq 40 \mathrm{mg}$ weekly lead to 2 CRs out of 26 evaluable AML patients [86].

Romidepsin is a potent, bicyclic tetrapeptide with DAC-inhibitory activity. Twenty patients with AML were treated at $13 \mathrm{mg} / \mathrm{m}(2) / \mathrm{d}$ on days 1,8 , and 15 of a 28 -day cycle. Antileukemic activity was observed in 5 of 7 patients with chromosomal abnormalities known to recruit HDACs, including those involving core binding factor (CBF). Two patients had clearance of bone marrow blasts and 3 patients had a greater than $50 \%$ decrease in bone marrow blasts. These responses were associated with a significant increase in MDR1, p15 and p14 expression [87]. In a phase II study in patients with high-risk MDS and AML, one patient achieved a CR, 6 patients experienced stable disease [88].

The hydroxamate DACi belinostat was administered as a 30-min i.v. infusion on days $1-5$ of a $21-d$ cycle to 16 patients with advanced hematologic malignancies. No complete or partial remissions were noted in these heavily pre-treated patients [89].

In contrast to the potent pan-DACi panobinstat, belinostat and vorinostat, the orally administered benzamide MGCD0103 is a selective inhibitor of HDAC1, 2, 3 (class $1)$ and 11 (class 4 ) and does not inhibit class 2 HDACs. In a phase I study, 3 of 29 patients with leukemia or MDS achieved a CR [90].

A phase I dose escalation study of the synthetic benzamide derivative MS-275 was completed in 75 patients with advanced acute leukemias. In spite of biologic alterations such as increase in protein and histone $\mathrm{H} 3 / \mathrm{H} 4$ acetylation, p21 expression, and caspase- 3 activation in bone marrow mononuclear cells, no clinical responses were seen [91].

\section{Toxicity of deacetylase inhibitors}

The most frequently adverse effects observed during DACi treatment for hematologic malignancies include gastrointestinal toxicity with nausea, vomiting and diarrhea, as well as fatigue, thrombocytopenia, neutropenia and non-specific ECG changes such as flattened T-waves, ST-segment depression, and QT-prolongation [92].

These changes are dose-dependent and represent a class effect of DACi. The pathogenesis of thrombocytopenia is unclear, but does not appear to involve cytotoxic mechanisms, as they are reversible within a few days of drug discontinuation. The other adverse events typically also rapidly revert to normal, or are easily controlled by prompt institution of supportive therapy, e.g. in the case of diarrhea. Nevertheless, the different DACi display some notable differences regarding their toxicity profiles.

\section{Epigenetic therapy combining deacetylase- and DNA-methyltransferase-inhibitors and/or ATRA in AML and MDS}

The first clinical studies to combine DACi and hypomethylating agents in patients with MDS and AML were conducted with VPA (see Table 1). Promising results were obtained in each of these studies [93-96] indicating both improved and accelerated responses with combined epigenetic therapy: the time to response was significantly shorter with combined therapy (1-3 cycles) than with single-agent DNMT (4-6 cycles). VPA was targeted to therapeutic plasma levels of at least $50 \mu \mathrm{g} / \mathrm{mL}$ to increase the efficacy of the hypomethylating agent [96], but no association between histone acetylation and response was observed [97]. Further correlative studies demonstrated reversal of p15 or CDH-1 promoter methylation during the first cycle of therapy in all six responding patients, whereas no demethylation was observed in any of the six nonresponders [97].

Even though these trials were designed primarily as dose-finding studies, the $20-30 \%$ remission rate (CR and $\mathrm{CRi}$ ) was higher than generally observed with 5 -azacitidine or decitabine when used as a single agent. This also holds true for a combination trial of SAHA and decitabine: of 61 patients evaluable for response, a CR or CRi was achieved by $18 \%$ patients with MDS, $8 \%$ with relapsed/refractory AML, and 36\% with untreated AML. [98] Even higher CR/ CRi rates of up to 40 to $60 \%$ were achieved in untreated patients with AML by combining a hypomethylating agent 
Table 1 Clinical trials evaluating DACi in combination with DNMT inhibitors

\begin{tabular}{|c|c|c|c|c|c|}
\hline & $\begin{array}{l}\text { VPA (d 1-10) + } \\
\text { Decitabine (d 1-10) [93], }\end{array}$ & $\begin{array}{l}\text { VPA (d 5-21) } \\
+ \text { Decitabine } \\
\text { (d 1-10) [94] }\end{array}$ & $\begin{array}{l}\text { VPA + Azacitidine (d 1- } \\
\text { 7) + ATRA (d 3-7) [95] }\end{array}$ & $\begin{array}{l}\text { VPA cont. + } \\
\text { Azaciditine } \\
\text { (d 1-7) [96] }\end{array}$ & $\begin{array}{l}\text { Phenylbutyrate ( } 7 \mathrm{~d} \text { cont.) }+ \\
\text { Azacitidine (d1-14 max.) [97] }\end{array}$ \\
\hline Patient numbers & $N=54$ & $N=25$ & $N=53$ & $N=62$ & $N=32$ \\
\hline Diagnosis & AML (89\%), MDS (11\%) & AML & AML (92\%) MDS (8\%) & MDS & AML (56\%) MDS (44\%) \\
\hline $\begin{array}{l}\text { Response (CR, } \\
\text { CRi) }\end{array}$ & $22 \%$ & $32 \%$ & $28 \%$ & $12 \%$ & $9 \%$ \\
\hline $\begin{array}{l}\text { No of previously } \\
\text { untreated patients }\end{array}$ & 11 & 12 & 33 & na & 8 \\
\hline $\begin{array}{l}\text { Response of previously } \\
\text { untreated patients }\end{array}$ & $50 \%$ & $58 \%$ & $42 \%$ & na & $25 \%$ \\
\hline $\begin{array}{l}\text { Survival of responding } \\
\text { patients }\end{array}$ & median 15 months & $3-10$ months & $>5$ months & na & $8-19+$ months \\
\hline
\end{tabular}

with VPA. A recent follow up of these untreated patients revealed that responders received more cycles of therapy and had significantly longer survival. Non-responding patients had a higher WBC and higher bone marrow blast count at the start of therapy. In general, patients who relapse after combination epigenetic therapy appeared to have a poor prognosis [99].

Numerous further combination studies using potent HDACi are currently being conducted, the results of which are being awaited with interest.

\section{Combination of deacetylase inhibitors with cytotoxic agents}

The rationale for phase I trials of the sequential combination of vorinostat followed by cytotoxic agents in patients with acute leukemias stems from in vitro studies showing that the sequence-dependent interaction of vorinostat and cytarabine or etoposide arrested the cells in G1 or G2 phase during vorinostat treatment and allowed recovery into $\mathrm{S}$ phase after removal of vorinostat [68]. Concurrent administration of vorinostat and idarubicin for 3 days was demonstrated in a phase I trial in 41 patients with refractory leukaemia (90\% AML), with $2 \mathrm{CRs}, 1 \mathrm{CRi}$ and 4 marrow responses. Correlative studies demonstrated histone acetylation in patients on therapy and modulation of CDKN1A and TOP2A (topoisomerase II) gene expression and a doserelated elevation in plasma vorinostat concentrations [100].

Preliminary results of a phase II study of vorinostat followed by idarubicin and cytarabine with 45 mostly highrisk AML patients aged $>65$ years were reported. Induction therapy consisted of oral vorinostat $500 \mathrm{mg}$ TID (days 1 to 3 ), idarubicin $12 \mathrm{mg} / \mathrm{m}^{2}$ iv (days 4 to 6) and cytarabine $\left(1.5 \mathrm{~g} / \mathrm{m}^{2}\right.$ as a continuous infusion on days 4 to 7$)$ followed by a maximum of 5 consolidation cycles with dose-reduced chemotherapy. Complete remission (CR) after one course of therapy was achieved in 35 patients and 1 patient achieved a $\mathrm{CRi}$ for an overall response rate of $80 \%$. No excess toxicity with the addition of vorinostat has been observed compared to standard induction therapy [101].

\section{DACi-associated drug resistance}

The rationale for developing deacetylase inhibitors as antileukemic agents is based on evidence showing induction of cellular differentiation, growth arrest, and apoptosis of malignant cells, but there is no evidence that only genes involved in leukemogenesis are targeted by DACi. Recent reports indicate that expression of the multidrug resistance1 (MDR1) gene is also regulated by epigenetic mechanisms, raising the possibility that this and other drug transporters able to counteract the cytotoxicity of various anti-leukemic drugs may be upregulated by DACi. This was in fact shown to be the case in a recent in vitro study demonstrating that in AML cells, expression of MDR1, breast cancer resistance protein (BCRP), and multidrug resistance-associated proteins (MRP) 7 and 8 were induced by phenylbutyrate, valproate, vorinostat or trichostatin $\mathrm{A}$ in a dose- and time-dependent manner. The pattern of gene induction by different DACi was shown to be cell line specific and associated with hyperacetylation of histone proteins in the promoter regions of MDR1, BCRP, and MRP8. Drug-induced apoptosis was impaired in KG- $1 \alpha$ cells treated with phenylbutyrate, resulting in resistance to daunorubicin, mitoxantrone, etoposide, vinblastine, paclitaxel, topotecan, gemcitabine, and 5-fluorouracil [102].

Similarly, the combination of ATRA with depsipeptide (FK228) induced MDR1 expression in NB4 promyelocytic leukemia (APL) cells, which normally do not express MDR1 and are highly sensitive to anthracyclines. Upregulation of MDR1 expression occurred via increased H4 and 
H3-Lys9 acetylation of the MDR1 promoter, and prevented doxorubicin-induced growth inhibition and apoptosis in APL cells. G1 cell-cycle arrest and upregulation of p21 mRNA may have further impaired the induction of apoptosis of cells in G2 phase. Conversely, initial exposure to doxorubicin followed by ATRA/FK228 treatment enhanced apoptosis [103]. These results indicate that epigenetic mechanisms leading to a drug resistance phenotype broader than the "classic multidrug resistance" may be activated by DACi exposure of AML cells, an effect which might impair therapeutic efficacy. This highlights the importance of investigating mechanism-based sequential therapies in clinical trials that combine DAC inhibitors with other agents commonly used for treatment of acute leukemias.

\section{DACi as treatment for acute leukemias and MDS: a critical appraisal and future perspectives}

Despite the profound preclinical activity of DACi against acute myeloid leukemia cells, their clinical development as treatment for myeloid malignancies has been less straightforward than for several lymphoid malignancies, most notably cutaneous $\mathrm{T}$ cell lymphoma. The to date modest success in AML and MDS highlights the limitations of our understanding of the complex interactions between epigenetic and genetic changes in these malignancies, and the paucity of our knowledge concerning the biological function of individual HDAC enzymes and the pleiotropic cellular effects of DACi. While it has been established that treatment of leukemic cells with DACi induces cell death, differentiation and/or cell-cycle arrest, they may also effect neoplastic growth by influencing the tumor microenvironment, regulating host immune responses or modifying the properties of normal hematopoietic cells. Thus, the beneficial effect of valproic acid in patients with low risk MDS may actually be a reflection of the unique ability of VPA to enhance normal hematopoietic function, whereas patients with high risk MDS or AML generally do not benefit because of the insufficient anti-leukemic activity of VPA in conjunction with the depletion of normal, potentially VPAresponsive progenitor cells.

In addition, the initial concept that DACi mediate their biological effects only through the regulation of gene expression via direct hyperacetylation of histones is no longer valid, following realisation that DACi acetylate diverse non-histone proteins, thereby regulating a broad range of cellular functions independent of transcriptional mechanisms. It is therefore not surprising that the actions of DACi are cell-context dependent, and will differ according to the differential expression and function of the individual HDACs in a given leukemia. HDAC enzyme expression is therefore likely to determine differential sensitivity to various types and dosages of DAC inhibitors, as well as their toxicity profile.

Limited clinical experience has so far demonstrated the relative safety of DACi currently in clinical testing, but adverse effects that become particularly relevant with longterm use have been noted, some of which, such as fatigue and diverse gastrointestinal complaints, appear to be class effects. Moreover, safety aspects will need to be reevaluated when DACi are combined with other anticancer agents, an obvious next step in their clinical development given available evidence that the clinical benefit derived from epigenetic and chromatin modifiers will be accrued when they are combined with chemotherapy or other targeted anti-leukemic agents. The potential of DACi to upregulate drug efflux pumps is a notable example for potentially detrimental drug-drug interactions, although there is as yet no evidence that this is clinically relevant. A rational selection of appropriate combination partners for DAC inhibitors will require an improved understanding of the specific epigenetic and genetic aberrations of each hematologic malignancy, which will have to be complemented by the identification of predictive biomarkers. If these challenges can be met, DAC inhibitors should develop into an important element of novel, targeted treatment strategies for leukemia.

Conflict of Interest Gesine Bug: Honoraria from Novartis for Advisory Boards and presentations; Celgene for presentations.

Oliver Ottman: Honoraria for Novartis Advisory Boards and presentations; Celgene for Advisory Boards.

Logistical support during submission of this article was provided by Springer Healthcare LLC. This support was funded by Novartis.

Open Access This article is distributed under the terms of the Creative Commons Attribution Noncommercial License which permits any noncommercial use, distribution, and reproduction in any medium, provided the original author(s) and source are credited.

\section{References}

1. Weichert W (2009) HDAC expression and clinical prognosis in human malignancies. Cancer Lett 280(2):168-176

2. Redner R, Wang J, Liu J (1999) Chromatin remodelling and leukemia: new therapeutic paradigms. Blood 94:417-428

3. Jones LK, Saha V (2002) Chromatin modification, leukaemia and implications for therapy. Br J Haematol 118(3):714-727

4. Marks P, Rifkind RA, Richon VM, Breslow R, Miller T, Kelly WK (2001) Histone deacetylases and cancer: causes and therapies. Nat Rev Cancer 1(3):194-202

5. Johnstone RW, Licht JD (2003) Histone deacetylase inhibitors in cancer therapy: is transcription the primary target? Cancer Cell 4 (1):13-18

6. Lindemann RK, Gabrielli B, Johnstone RW (2004) Histonedeacetylase inhibitors for the treatment of cancer. Cell Cycle 3 (6):779-788 
7. Scandura JM, Boccuni P, Cammenga J, Nimer SD (2002) Transcription factor fusions in acute leukemia: variations on a theme. Oncogene 21(21):3422-3444

8. Durst KL, Hiebert SW (2004) Role of RUNX family members in transcriptional repression and gene silencing. Oncogene 23 (24):4220-4224

9. So CW, Cleary ML (2004) Dimerization: a versatile switch for oncogenesis. Blood 104(4):919-922

10. Borrow J, Stanton VP Jr, Andresen JM, Becher R, Behm FG, Chaganti RS et al (1996) The translocation $\mathrm{t}(8 ; 16)(\mathrm{p} 11 ; \mathrm{p} 13)$ of acute myeloid leukaemia fuses a putative acetyltransferase to the CREB-binding protein. Nat Genet 14(1):33-41

11. Sobulo OM, Borrow J, Tomek R, Reshmi S, Harden A, Schlegelberger B et al (1997) MLL is fused to CBP, a histone acetyltransferase, in therapy-related acute myeloid leukemia with at (11;16)(q23;p13.3). Proc Natl Acad Sci USA 94(16):8732-8737

12. Linggi B, Muller-Tidow C, van de Locht L, Hu M, Nip J, Serve $\mathrm{H}$ et al (2002) The $\mathrm{t}(8 ; 21)$ fusion protein, AML1 ETO, specifically represses the transcription of the p14(ARF) tumor suppressor in acute myeloid leukemia. Nat Med 8(7):743-750

13. Krug U, Ganser A, Koeffler HP (2002) Tumor suppressor genes in normal and malignant hematopoiesis. Oncogene 21(21):3475-3495

14. Liu S, Shen T, Huynh L, Klisovic MI, Rush LJ, Ford JL et al (2005) Interplay of RUNX1/MTG8 and DNA methyltransferase 1 in acute myeloid leukemia. Cancer Res 65(4):1277-1284

15. Yang XJ, Ullah M (2007) MOZ and MORF, two large MYSTic HATs in normal and cancer stem cells. Oncogene 26(37):54085419

16. Rebel VI, Kung AL, Tanner EA, Yang H, Bronson RT, Livingston DM (2002) Distinct roles for CREB-binding protein and p300 in hematopoietic stem cell self-renewal. Proc Natl Acad Sci USA 99(23):14789-14794

17. Gayther SA, Batley SJ, Linger L, Bannister A, Thorpe K, Chin SF et al (2000) Mutations truncating the EP300 acetylase in human cancers. Nat Genet 24(3):300-303

18. Yang XJ (2004) The diverse superfamily of lysine acetyltransferases and their roles in leukemia and other diseases. Nucleic Acids Res 32(3):959-976

19. Blobel GA (2000) CREB-binding protein and p300: molecular integrators of hematopoietic transcription. Blood 95(3):745-755

20. Rowley JD, Reshmi S, Sobulo O, Musvee T, Anastasi J, Raimondi $\mathrm{S}$ et al (1997) All patients with the $\mathrm{T}(11 ; 16)(\mathrm{q} 23 ; \mathrm{p} 13.3)$ that involves MLL and CBP have treatment-related hematologic disorders. Blood 90(2):535-541

21. Pui CH, Relling MV, Downing JR (2004) Acute lymphoblastic leukemia. N Engl J Med 350(15):1535-1548

22. Zelent A, Greaves M, Enver T (2004) Role of the TEL-AML1 fusion gene in the molecular pathogenesis of childhood acute lymphoblastic leukaemia. Oncogene 23(24):4275-4283

23. Agirre X, Vilas-Zornoza A, Jimenez-Velasco A, Martin-Subero JI, Cordeu L, Garate L et al (2009) Epigenetic silencing of the tumor suppressor microRNA Hsa-miR-124a regulates CDK6 expression and confers a poor prognosis in acute lymphoblastic leukemia. Cancer Res 69(10):4443-4453

24. Marchion D, Munster P (2007) Development of histone deacetylase inhibitors for cancer treatment. Expert Rev Anticancer Ther 7(4):583-598

25. Dokmanovic M, Marks PA (2005) Prospects: histone deacetylase inhibitors. J Cell Biochem 96(2):293-304

26. Gray SG, Qian CN, Furge K, Guo X, Teh BT (2004) Microarray profiling of the effects of histone deacetylase inhibitors on gene expression in cancer cell lines. Int J Oncol 24(4):773-795

27. Peart MJ, Smyth GK, van Laar RK, Bowtell DD, Richon VM, Marks PA et al (2005) Identification and functional significance of genes regulated by structurally different histone deacetylase inhibitors. Proc Natl Acad Sci USA 102(10):3697-3702
28. Bhalla KN (2005) Epigenetic and chromatin modifiers as targeted therapy of hematologic malignancies. J Clin Oncol 23 (17):3971-3993

29. Haggarty SJ, Koeller KM, Wong JC, Grozinger CM, Schreiber SL (2003) Domain-selective small-molecule inhibitor of histone deacetylase 6 (HDAC6)-mediated tubulin deacetylation. Proc Natl Acad Sci USA 100(8):4389-4394

30. Wolf D, Rodova M, Miska EA, Calvet JP, Kouzarides T (2002) Acetylation of beta-catenin by CREB-binding protein (CBP). J Biol Chem 277(28):25562-25567

31. Bannister AJ, Miska EA, Gorlich D, Kouzarides T (2000) Acetylation of importin-alpha nuclear import factors by $\mathrm{CBP} /$ p300. Curr Biol 10(8):467-470

32. Blander G, Zalle N, Daniely Y, Taplick J, Gray MD, Oren M (2002) DNA damage-induced translocation of the Werner helicase is regulated by acetylation. J Biol Chem 277(52):50934-50940

33. Cohen HY, Lavu S, Bitterman KJ, Hekking B, Imahiyerobo TA, Miller $\mathrm{C}$ et al (2004) Acetylation of the $\mathrm{C}$ terminus of Ku70 by $\mathrm{CBP}$ and PCAF controls Bax-mediated apoptosis. Mol Cell 13 (5):627-638

34. Fuino L, Bali P, Wittmann S, Donapaty S, Guo F, Yamaguchi H et al (2003) Histone deacetylase inhibitor LAQ824 downregulates Her-2 and sensitizes human breast cancer cells to trastuzumab, taxotere, gemcitabine, and epothilone B. Mol Cancer Ther 2(10):971-984

35. Hubbert C, Guardiola A, Shao R, Kawaguchi Y, Ito A, Nixon A et al (2002) HDAC6 is a microtubule-associated deacetylase. Nature 417(6887):455-458

36. Polevoda B, Sherman F (2002) The diversity of acetylated proteins. Genome Biol 3(5):reviews0006

37. Minucci S, Pelicci PG (2006) Histone deacetylase inhibitors and the promise of epigenetic (and more) treatments for cancer. Nat Rev Cancer 6(1):38-51

38. Glozak MA, Sengupta N, Zhang X, Seto E (2005) Acetylation and deacetylation of non-histone proteins. Gene 363:15-23

39. Insinga A, Monestiroli S, Ronzoni S, Gelmetti V, Marchesi F, Viale A et al (2005) Inhibitors of histone deacetylases induce tumor-selective apoptosis through activation of the death receptor pathway. Nature Med 11(1):71-76

40. Nebbioso A, Clarke N, Voltz E, Germain E, Ambrosino C, Bontempo $\mathrm{P}$ et al (2005) Tumor-selective action of HDAC inhibitors involves TRAIL induction in acute myeloid leukemia cells. Nature Med 11(1):77-84

41. Reikvam H, Ersvaer E, Bruserud O (2009) Heat shock protein 90 - a potential target in the treatment of human acute myelogenous leukemia. Curr Cancer Drug Targets 9(6):761-776

42. Göttlicher M, Minucci S, Zhu J, Krämer O, Schimpf A, Giavara $S$ et al (2001) Valproic acid defines a novel class of HDAC inhibitors inducing differentiation of transformed cells. EMBO J 20:6969-6978

43. Liu S, Klisovic RB, Vukosavljevic T, Yu J, Paschka P, Huynh L et al (2007) Targeting AML1/ETO-histone deacetylase repressor complex: a novel mechanism for valproic acid-mediated gene expression and cellular differentiation in AML1/ETO-positive acute myeloid leukemia cells. J Pharmacol Exp Ther 321 (3):953-960

44. Bug G, Schwarz K, Schoch C, Kampfmann M, Henschler R, Hoelzer D et al (2007) Effect of histone deacetylase inhibitor valproic acid on progenitor cells of acute myeloid leukemia. Haematologica 92(4):542-545

45. Barbetti V, Gozzini A, Rovida E, Morandi A, Spinelli E, Fossati $\mathrm{G}$ et al (2008) Selective anti-leukaemic activity of low-dose histone deacetylase inhibitor ITF2357 on AML1/ETO-positive cells. Oncogene 27(12):1767-1778

46. Murata M, Towatari M, Kosugi H, Tanimoto M, Ueda R, Saito H et al (2000) Apoptotic cytotoxic effects of a histone deacetylase 
inhibitor, FK228, on malignant lymphoid cells. Jpn J Cancer Res 91(11):1154-1160

47. Romanski A, Bacic B, Bug G, Pfeifer H, Gul H, Remiszewski S et al (2004) Use of a novel histone deacetylase inhibitor to induce apoptosis in cell lines of acute lymphoblastic leukemia. Haematologica 89(4):419-426

48. Scuto A, Kirschbaum M, Kowolik C, Kretzner L, Juhasz A, Atadja $P$ et al (2008) The novel histone deacetylase inhibitor, LBH589, induces expression of DNA damage response genes and apoptosis in Ph-acute lymphoblastic leukemia cells. Blood 111(10):5093-5100

49. Tsapis M, Lieb M, Manzo F, Shankaranarayanan P, Herbrecht R, Lutz P et al (2007) HDAC inhibitors induce apoptosis in glucocorticoid-resistant acute lymphatic leukemia cells despite a switch from the extrinsic to the intrinsic death pathway. Int $\mathrm{J}$ Biochem Cell Biol 39(7-8):1500-1509

50. Stams WA, den Boer ML, Beverloo HB, Kazemier KM, van Wering ER, Janka-Schaub GE et al (2005) Effect of the histone deacetylase inhibitor depsipeptide on B-cell differentiation in both TEL-AML1-positive and negative childhood acute lymphoblastic leukemia. Haematologica 90(12):1697-1699

51. Einsiedel HG, Kawan L, Eckert C, Witt O, Fichtner I, Henze G et al (2006) Histone deacetylase inhibitors have antitumor activity in two NOD/SCID mouse models of B-cell precursor childhood acute lymphoblastic leukemia. Leukemia 20(8):1435-1436

52. Richon VM, Webb Y, Merger R, Sheppard T, Jursic B, Ngo L et al (1996) Second generation hybrid polar compounds are potent inducers of transformed cell differentiation. Proc Natl Acad Sci USA 93(12):5705-5708

53. Archer SY, Meng S, Shei A, Hodin RA (1998) p21(WAF1) is required for butyrate-mediated growth inhibition of human colon cancer cells. Proc Natl Acad Sci USA 95(12):67916796

54. He LZ, Tolentino T, Grayson P, Zhong S, Warrell RP Jr, Rifkind RA et al (2001) Histone deacetylase inhibitors induce remission in transgenic models of therapy-resistant acute promyelocytic leukemia. J Clin Investig 108(9):1321-1330

55. Ferrara F, Fazi F, Bianchini A, Padula F, Gelmetti V, Minucci S et al (2001) Histone deacytylase-targeted treatment restores retinoic acid signaling and differentiation in acute myeloid leukemia. Cancer Res 61:2-7

56. Cameron EE, Bachman KE, Myohanen S, Herman JG, Baylin SB (1999) Synergy of demethylation and histone deacetylase inhibition in the re-expression of genes silenced in cancer. Nat Genet 21(1):103-107

57. Bolden JE, Peart MJ, Johnstone RW (2006) Anticancer activities of histone deacetylase inhibitors. Nat reviews 5(9):769-784

58. Fabre C, Grosjean J, Tailler M, Boehrer S, Ades L, Perfettini JL et al (2008) A novel effect of DNA methyltransferase and histone deacetylase inhibitors: NFkappaB inhibition in malignant myeloblasts. Cell Cycle 7(14):2139-2145

59. Fiskus W, Buckley K, Rao R, Mandawat A, Yang Y, Joshi R et al (2009) Panobinostat treatment depletes EZH2 and DNMT1 levels and enhances decitabine mediated de-repression of JunB and loss of survival of human acute leukemia cells. Cancer Biol Ther 8(10):939-950

60. Huang Y, Waxman S (1998) Enhanced growth inhibition and differentiation of fluorodeoxyuridine-treated human colon carcinoma cells by phenylbutyrate. Clin Cancer Res 4(10):2503-2509

61. Kim MS, Blake M, Baek JH, Kohlhagen G, Pommier Y, Carrier F (2003) Inhibition of histone deacetylase increases cytotoxicity to anticancer drugs targeting DNA. Cancer Res 63(21):72917300

62. Mitsiades CS, Mitsiades NS, McMullan CJ, Poulaki V, Shringarpure R, Hideshima T et al (2004) Transcriptional signature of histone deacetylase inhibition in multiple myeloma: biological and clinical implications. Proc Natl Acad Sci USA 101(2):540-545

63. Witzig TE, Timm M, Stenson M, Svingen PA, Kaufmann SH (2000) Induction of apoptosis in malignant B cells by phenylbutyrate or phenylacetate in combination with chemotherapeutic agents. Clin Cancer Res 6(2):681-692

64. Marchion DC, Bicaku E, Daud AI, Richon V, Sullivan DM, Munster PN (2004) Sequence-specific potentiation of topoisomerase II inhibitors by the histone deacetylase inhibitor suberoylanilide hydroxamic acid. J Cell Biochem 92(2):223-237

65. Kurz EU, Wilson SE, Leader KB, Sampey BP, Allan WP, Yalowich JC et al (2001) The histone deacetylase inhibitor sodium butyrate induces DNA topoisomerase II alpha expression and confers hypersensitivity to etoposide in human leukemic cell lines. Mol Cancer Ther 1(2):121-131

66. Sanchez-Gonzalez B, Yang H, Bueso-Ramos C, Hoshino K, Quintas-Cardama A, Richon VM et al (2006) Antileukemia activity of the combination of an anthracycline with a histone deacetylase inhibitor. Blood 108(4):1174-1182

67. Maiso P, Colado E, Ocio EM, Garayoa M, Martin J, Atadja P et al (2009) The synergy of panobinostat plus doxorubicin in acute myeloid leukemia suggests a role for HDAC inhibitors in the control of DNA repair. Leukemia 23(12):2265-2274

68. Shiozawa K, Nakanishi T, Tan M, Fang HB, Wang WC, Edelman MJ et al (2009) Preclinical studies of vorinostat (suberoylanilide hydroxamic acid) combined with cytosine arabinoside and etoposide for treatment of acute leukemias. Clin Cancer Res 15(5):1698-1707

69. Maggio SC, Rosato RR, Kramer LB, Dai Y, Rahmani M, Paik DS et al (2004) The histone deacetylase inhibitor MS-275 interacts synergistically with fludarabine to induce apoptosis in human leukemia cells. Cancer Res 64(7):2590-2600

70. Leclerc GJ, Mou C, Leclerc GM, Mian AM, Barredo JC (2010) Histone deacetylase inhibitors induce FPGS mRNA expression and intracellular accumulation of long-chain methotrexate polyglutamates in childhood acute lymphoblastic leukemia: implications for combination therapy. Leukemia 24(3):552-562

71. Bali P, George P, Cohen P, Tao J, Guo F, Sigua C et al (2004) Superior activity of the combination of histone deacetylase inhibitor LAQ824 and the FLT-3 kinase inhibitor PKC412 against human acute myelogenous leukemia cells with mutant FLT-3. Clin Cancer Res 10(15):4991-4997

72. Nishioka C, Ikezoe T, Yang J, Koeffler HP, Yokoyama A (2008) Blockade of mTOR signaling potentiates the ability of histone deacetylase inhibitor to induce growth arrest and differentiation of acute myelogenous leukemia cells. Leukemia 22(12):2159-2168

73. Kircher B, Schumacher P, Petzer A, Hoflehner E, Haun M, Wolf AM et al (2009) Anti-leukemic activity of valproic acid and imatinib mesylate on human $\mathrm{Ph}+\mathrm{ALL}$ and CML cells in vitro. Eur J Haematol 83(1):48-56

74. Miller CP, Ban K, Dujka ME, McConkey DJ, Munsell M, Palladino M et al (2007) NPI-0052, a novel proteasome inhibitor, induces caspase- 8 and ROS-dependent apoptosis alone and in combination with HDAC inhibitors in leukemia cells. Blood 110 (1):267-277

75. Miller CP, Rudra S, Keating MJ, Wierda WG, Palladino M, Chandra J (2009) Caspase-8 dependent histone acetylation by a novel proteasome inhibitor, NPI-0052: a mechanism for synergy in leukemia cells. Blood 113(18):4289-4299

76. Kuendgen A, Strupp C, Aivado M, Bernhardt A, Hildebrandt B, Haas R, et al (2004) Treatment of myelodysplastic syndromes with valproic acid alone or in combination with all-trans retinoic acid. Blood. May 20

77. Kuendgen A, Knipp S, Fox F, Strupp C, Hildebrandt B, Steidl C et al (2005) Results of a phase 2 study of valproic acid alone or in combination with all-trans retinoic acid in 75 patients with 
myelodysplastic syndrome and relapsed or refractory acute myeloid leukemia. Ann Hematol 84(Suppl 13):61-66

78. Kuendgen A, Gattermann N (2007) Valproic acid for the treatment of myeloid malignancies. Cancer 110(5):943-954

79. Kuendgen A, Schmid M, Schlenk R, Knipp S, Hildebrandt B, Steidl C et al (2006) The histone deacetylase (HDAC) inhibitor valproic acid as monotherapy or in combination with all-trans retinoic acid in patients with acute myeloid leukemia. Cancer 106(1):112-119

80. Bug G, Ritter M, Wassmann B, Schoch C, Heinzel T, Schwarz K et al (2005) Clinical trial of valproic acid and all-trans retinoic acid in patients with poor-risk acute myeloid leukemia. Cancer 104(12):2717-2725

81. Cimino G, Lo-Coco F, Fenu S, Travaglini L, Finolezzi E, Mancini M et al (2006) Sequential valproic acid/all-trans retinoic acid treatment reprograms differentiation in refractory and highrisk acute myeloid leukemia. Cancer Res 66(17):8903-8911

82. Raffoux E, Chaibi P, Dombret H, Degos L (2005) Valproic acid and all-trans retinoic acid for the treatment of elderly patients with acute myeloid leukemia. Haematologica 90(7):986-988

83. Garcia-Manero G, Yang H, Bueso-Ramos C, Ferrajoli A, Cortes J, Wierda WG et al (2008) Phase 1 study of the histone deacetylase inhibitor vorinostat (suberoylanilide hydroxamic acid [SAHA]) in patients with advanced leukemias and myelodysplastic syndromes. Blood 111(3):1060-1066

84. Schaefer EW, Loaiza-Bonilla A, Juckett M, DiPersio JF, Roy V, Slack J et al (2009) A phase 2 study of vorinostat in acute myeloid leukemia. Haematologica 94(10):1375-1382

85. Giles F, Fischer T, Cortes J, Garcia-Manero G, Beck J, Ravandi F et al (2006) A phase I study of intravenous LBH589, a novel cinnamic hydroxamic acid analogue histone deacetylase inhibitor, in patients with refractory hematologic malignancies. Clin Cancer Res 12(15):4628-4635

86. Ottmann O, Spencer A, Prince H, Bhalla K, Fischer T, Liu A et al (2008) Phase IA/II study of oral panobinostat (LBH589), a novel pan-deacetylase inhibitor (DACi) demonstrating efficacy in patients with advanced hematologic malignancies. Blood 112:a958

87. Odenike OM, Alkan S, Sher D, Godwin JE, Huo D, Brandt SJ et al (2008) Histone deacetylase inhibitor romidepsin has differential activity in core binding factor acute myeloid leukemia. Clin Cancer Res 14(21):7095-7101

88. Klimek VM, Fircanis S, Maslak P, Guernah I, Baum M, Wu N et al (2008) Tolerability, pharmacodynamics, and pharmacokinetics studies of depsipeptide (romidepsin) in patients with acute myelogenous leukemia or advanced myelodysplastic syndromes. Clin Cancer Res 14(3):826-832

89. Gimsing P, Hansen M, Knudsen LM, Knoblauch P, Christensen IJ, Ooi CE et al (2008) A phase I clinical trial of the histone deacetylase inhibitor belinostat in patients with advanced hematological neoplasia. Eur J Haematol 81(3):170-176

90. Garcia-Manero G, Assouline S, Cortes J, Estrov Z, Kantarjian H, Yang $H$ et al (2008) Phase 1 study of the oral isotype specific histone deacetylase inhibitor MGCD0103 in leukemia. Blood 112(4):981-989

91. Gojo I, Jiemjit A, Trepel JB, Sparreboom A, Figg WD, Rollins S et al (2007) Phase 1 and pharmacologic study of MS-275, a histone deacetylase inhibitor, in adults with refractory and relapsed acute leukemias. Blood 109(7):2781-2790

92. Piekarz RL, Frye AR, Wright JJ, Steinberg SM, Liewehr DJ, Rosing DR et al (2006) Cardiac studies in patients treated with depsipeptide, FK228, in a phase II trial for T-cell lymphoma. Clin Cancer Res 12(12):3762-3773

93. Garcia-Manero G, Kantarjian HM, Sanchez-Gonzalez B, Yang H, Rosner G, Verstovsek S, et al (2006) Phase I/II study of the combination of 5-aza-2'-deoxycytidine with valproic acid in patients with leukemia. Blood. Aug 1

94. Blum W, Klisovic RB, Hackanson B, Liu Z, Liu S, Devine H et al (2007) Phase I study of decitabine alone or in combination with valproic acid in acute myeloid leukemia. J Clin Oncol 25 (25):3884-3891

95. Soriano AO, Yang H, Faderl S, Estrov Z, Giles F, Ravandi F et al (2007) Safety and clinical activity of the combination of 5azacytidine, valproic acid, and all-trans retinoic acid in acute myeloid leukemia and myelodysplastic syndrome. Blood 110(7):2302-2308

96. Voso MT, Santini V, Finelli C, Musto P, Pogliani E, Angelucci E et al (2009) Valproic acid at therapeutic plasma levels may increase 5-azacytidine efficacy in higher risk myelodysplastic syndromes. Clin Cancer Res 15(15):5002-5007

97. Gore SD, Baylin S, Sugar E, Carraway H, Miller CB, Carducci $\mathrm{M}$ et al (2006) Combined DNA methyltransferase and histone deacetylase inhibition in the treatment of myeloid neoplasms. Cancer Res 66(12):6361-6369

98. Kirschbaum M, Gojo I, Goldberg S, Kujawski L, Atallah E, Marks PA et al (2009) Vorinostat in combination with decitabine for the treatment of relapsed or newly diagnosed Acute Myelogenous Leukemia (AML) or Myelodysplastic Syndrome (MDS): a phase i, dose-escalation study. Blood 114:2089a

99. Kadia T, Estrov Z, Ravandi F, Koller C, Borthakur G, Jabbour E et al (2009) Long term followup and patterns of failure in patients with Acute Myeloid Leukemia (AML) and high risk Myelodysplastic Syndrome (MDS) treated on studies combining a hypomethylating agent and the histone deacetylase inhibitor (HDACi) valproic acid. Blood 114:2074a

100. Kadia TM, Yang H, Ferrajoli A, Maddipotti S, Schroeder C, Madden TL, et al (2010) A phase I study of vorinostat in combination with idarubicin in relapsed or refractory leukaemia. Br J Haematol. Apr 29

101. Garcia-Manero G, Tambaro F, Bekele B, Jabbour E, Ravandi F, Yang $\mathrm{H}$ et al (2009) Phase II study of vorinostat in combination with idarubicin (Ida) and cytarabine (ara-C) as front line therapy in Acute Myelogenous Leukemia (AML) or higher risk myelodysplastic syndrome (MDS). Blood 114:1055a

102. Hauswald S, Duque-Afonso J, Wagner MM, Schertl FM, Lubbert M, Peschel C et al (2009) Histone deacetylase inhibitors induce a very broad, pleiotropic anticancer drug resistance phenotype in acute myeloid leukemia cells by modulation of multiple $\mathrm{ABC}$ transporter genes. Clin Cancer Res 15(11):3705-3715

103. Tabe Y, Konopleva M, Contractor R, Munsell M, Schober WD, Jin L et al (2006) Up-regulation of MDR1 and induction of doxorubicin resistance by histone deacetylase inhibitor depsipeptide (FK228) and ATRA in acute promyelocytic leukemia cells. Blood 107(4):1546-1554 